\title{
Thyroid Storm: A Japanese Perspective
}

\author{
Takashi Akamizu
}

Background: Thyroid storm (TS) is life threatening. In the mid-2000s, its incidence was poorly defined, peerreviewed diagnostic criteria were not available, and management and treatment did not seem to be verified based upon evidence and latest advances in medicine.

Methods: First, diagnostic criteria were developed based on 99 patients in the literature and seven patients in this study. Then, initial and follow-up surveys were conducted from 2004 through 2008, targeting all hospitals in Japan to obtain and verify information on patients who met diagnostic criteria for TS. Based on these data, the diagnostic criteria were revised, and management and treatment guidelines were created.

Results: The incidence of TS in hospitalized patients in Japan was estimated to be 0.20 per 100,000 per year and $0.22 \%$ of all thyrotoxic patients. The mortality rate was $10.7 \%$. Multiple organ failure was the most common cause of death, followed by congestive heart failure, respiratory failure, and arrhythmia. In the final diagnostic criteria for TS, the definition of jaundice as serum bilirubin concentration $>3 \mathrm{mg} / \mathrm{dL}$ was added. Based upon nationwide surveys and the latest information, guidelines for the management and treatment for TS were extensively revised and algorithms were developed.

Conclusions: TS remains a life-threatening disorder, with $>10 \%$ mortality in Japan. New peer-reviewed diagnostic criteria for TS are presented and its clinical features, prognosis, and incidence are clarified based on nationwide surveys. Furthermore, this information helped to establish detailed guidelines for the management and treatment of TS. A prospective prognostic study to validate the guidelines is eagerly anticipated.

Keywords: thyroid crisis, guideline, diagnostic criteria, treatment, management

\section{Introduction}

$\mathbf{T}^{\mathrm{s}}$ HE TERM “THYROID STORM" (TS) or crisis appeared in the literature in the late 1920s. For example, in 1928, Lahey gave this early description: "The following remarks concerning thyroid crises and their management are prompted by the fact that so many patients are constantly being brought to the hospital in advanced stages of thyroid crisis and because so many of these inoperable patients die in spite of employment of every measure with which we are acquainted" $(1,2)$. At that time, the principal measures to manage this condition were iodine, fluids, glucose, and morphine. Out of approximately 550 patients who underwent thyroid surgery for hyperthyroidism, six died of thyroid crisis (1). Other terms such as "acute hyperthyroidism" and "thyroid delirium" are found in the literature from those days. Since TS used to occur most often after thyroidectomy, it was called "surgical crisis" to differentiate it from nonsurgical or medical crisis $(3,4)$.
In the 1940s, the introduction of radioactive iodine therapy and antithyroid drugs (ATDs) fundamentally changed the treatment of hyperthyroidism (5). In particular, the treatment of Graves' disease (GD) with ATDs made it possible for patients to maintain a euthyroid state before thyroidectomy. Thyroid surgery in the euthyroid state is much safer than in the thyrotoxic state, resulting in a marked decrease in the occurrence of surgical crisis $(3,4)$. The incidence of medical crisis was also thought to have decreased as a result of the emergence of radioactive iodine therapy and ATDs.

In the mid-2000s, clinical thyroid specialists rarely encountered TS, if at all, in Japan. The more specialized a clinician was in the field of thyroid medicine, the less experience he or she had with TS. Specialized clinicians, particularly in thyroid clinics or hospitals, usually saw typical thyroid patients but no emergency cases. More than a few famous thyroidologists have said, "Thyroid storm has been extinguished in Japan." However, some endocrinologists in large hospitals with emergency departments have encountered patients with

\footnotetext{
The First Department of Medicine, Wakayama Medical University, Wakayama, Japan.
}

(c) Takashi Akamizu 2018; Published by Mary Ann Liebert, Inc. This is an Open Access article distributed under the terms of the Creative Commons Attribution License, which permits unrestricted use, distribution, and reproduction in any medium, provided the original work is properly cited. 
TS, albeit not so often, thus raising the concern that some patients with TS or impending TS might be overlooked or misdiagnosed. At that time, there was no evidence-based epidemiological data on TS. One report suggested that TS might account for no more than $1-2 \%$ of hospital admissions for thyrotoxicosis (6-8). Moreover, no peer-reviewed diagnostic criteria have been reported. Few studies have been published, other than those by Burch and Wartofsky (9). Even concerning management and treatment of TS, descriptions in textbooks did not seem to provide enough information to save patients with life-threatening TS, and there have been no updated guidelines for this disorder. In this context, in 2006, the Japan Thyroid Association (JTA) organized a committee to develop diagnostic criteria for TS and performed a survey to assess its incidence in Japan, linking the research activities of the Japan Endocrine Society and the Ministry of Health, Labor, and Welfare of Japan. In 2012, as the members of this committee, findings were reported regarding the epidemiological data and clinical features of TS, and diagnostic criteria for TS were proposed (10). As the next step, based on the evidence from nationwide surveys and additional literature reviews, guidelines for the management of TS were established in 2016 $(11,12)$. The guidelines include 15 recommendations for the treatment of thyrotoxicosis and central nervous system, cardiovascular system, and hepato-gastrointestinal tract failure; criteria for admission to the intensive care unit; and prognostic evaluation. Approaches to prevent TS, the role of definitive therapy, and plans for future prospective trials for the treatment of TS were also proposed.

This review outlines our clinical studies on TS for approximately a decade (10-12). In addition, some intriguing data that were not included in published manuscripts due to space limitations are introduced. It is hoped that this effort will be useful to many physicians in Japan and all over the world for the management of patients with TS and that it will contribute to improvements in outcomes.

\section{Definition of TS}

TS is a life-threatening condition requiring emergency treatment. The condition, which is often triggered by severe physical or mental stress, arises in thyrotoxic patients. These patients manifest multiple organ failure as a result of the breakdown of compensatory mechanisms (Table 1) (10). Because the pathophysiological mechanism of TS has not been clarified, TS is defined clinically.

\section{Epidemiology of TS}

Until the mid-2000s, the incidence of TS had not been fully investigated in a large population. Only one report could be found to indicate that TS might account for no more than 1-2\% of hospital admissions for thyrotoxicosis (6-8). The mortality rate was very variable, ranging from $10 \%$ to $75 \%$. Thus, the author attempted to investigate TS $(4,8,9,13)$.

Nationwide surveys were conducted from 2004 through 2008 to obtain a five-year estimate of TS incidence in Japan (Table 2) (10,12). Before the surveys, the tentative first edition of diagnostic criteria, as described below in the Diagnosis section, was created. The incidence rate was estimated to be 0.20 persons per 100,000 population per year in Japan, accounting for $0.22 \%$ of all thyrotoxic patients and $5.4 \%$ of hospitalized thyrotoxic patients. The incidence gradually increased in the last two years (2007-2008) in Endocrinology Units but not in Emergency Units (Fig. 1). This may be partly due to increased awareness of TS because research activities had often been presented at endocrine or thyroid meetings since the committee was founded in 2006. GD was the most common cause of thyrotoxicosis among patients with TS, followed by very rare cases of destructive thyroiditis. In $45 \%$ of cases, the duration between TS onset and the initial diagnosis of GD was less than one year. Approximately $20 \%$ of patients developed TS before they received ATD treatment. Factors classically considered to trigger TS were present in $70 \%$ of patients with TS. The most common trigger of TS was the irregular use or discontinuation of antithyroid medication, and the second most common trigger was infection, particularly of the upper respiratory tract. The mortality rate of patients with definite or suspected TS was $10.7 \%$ (38/356). Individually, the mortality rates for definite and suspected cases were $11.0 \%$ and $9.5 \%$, respectively (10).

The most common causes of death in the 356 patients with TS were multiple organ failure (MOF) and congestive heart failure (CHF; Fig. 2) (10). In a simple regression analysis, many factors including Glasgow Coma Scale (GCS; odds ratio $[\mathrm{OR}]=0.863 ; p=0.0016), \mathrm{CHF}$ as a comorbidity $(\mathrm{OR}=2.086 ; p=0.0426)$, and serum creatinine $(\mathrm{OR}=2.535$; $p=0.0025)$ were significantly associated with mortality. However, multiple logistic regression revealed that the comorbidities of shock $(\mathrm{OR}=3.90 ; p=0.006)$, disseminated intravascular coagulation (DIC; $\mathrm{OR}=3.91 ; p=0.012$ ), and MOF $(\mathrm{OR}=9.85 ; p<0.001)$ were independent prognostic factors for death. Among the survivors, 22 were reported as having irreversible damage of some kind, with brain damage occurring in six patients, disuse atrophy occurring in five, cerebrovascular disease occurring in four, renal insufficiency occurring in two, and psychosis occurring in two. In three patients, the type of irreversible damage was not specified. Higher GCS and elevated blood urea nitrogen (BUN) were significantly associated with the development of irreversible deficits (GCS: $\mathrm{OR}=0.846, p=0.0062$; $\mathrm{BUN}$ : $\mathrm{OR}=1.01$, $p=0.0434)$.

\section{Pathophysiology of TS}

The pathogenesis of TS has not been fully clarified. Most studies have found no difference in thyroid hormone levels between patients with TS and uncomplicated thyrotoxicosis $(4,9)$, including the author's study $(10,11)$. Nonetheless, thyrotoxicosis is essential for the pathogenesis of TS because TS has not been reported in Graves' patients who underwent thyroidectomy with well-controlled thyroid function (10). On the other hand, there were six cases of TS in patients who underwent radioiodine treatment, suggesting that an acute increase in thyroxine (T4) or triiodothyronine (T3) release from the thyroid plays a role in the development of TS (10).

The most common illness that precipitates TS is infection, as observed in the author's study $(8,10)$. Inflammatory responses induce cytokines such as tumor necrosis factor alpha $(\mathrm{TNF}-\alpha)$, interleukin 1 , and interleukin 6. Cytokines can affect the expression of many proteins involved in thyroid hormone metabolism (14). Inflammatory signaling pathways such as nuclear factor kappa-light-chain-enhancer of activated $\mathrm{B}$ cells $(\mathrm{NF} \kappa \mathrm{B})$ and activator protein 1 might be activated. These responses may induce changes in enzymes 


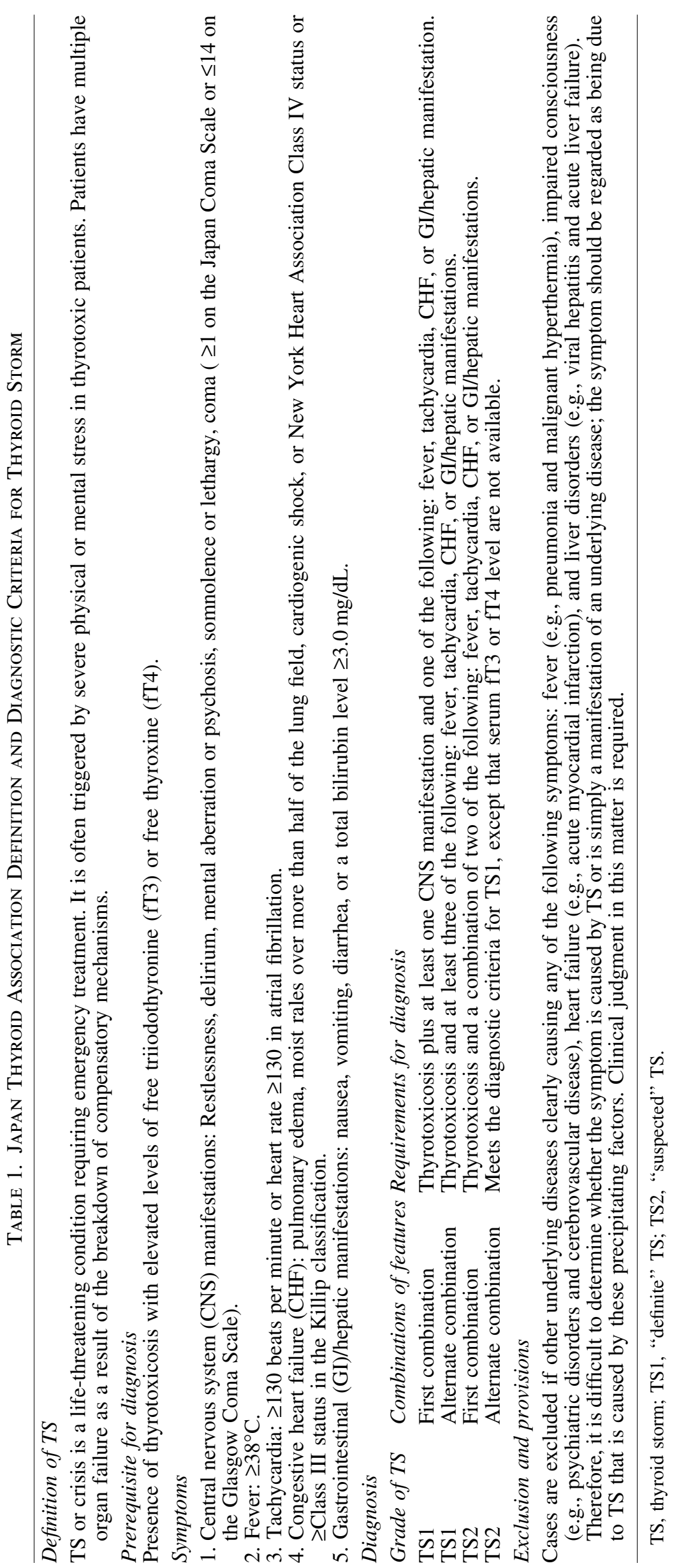


Table 2. Summary of Nationwide Surveys in Japan

- Tentative diagnostic criteria, based mainly on patients in the literature, were initially developed for nationwide surveys of thyroid storm (TS).

- Nationwide surveys in the period from 2004 to 2008 were conducted in 2009 to obtain epidemiological data, including a five-year estimate of TS incidence in Japan, clinical features, and prognosis.

- The incidence rate is estimated to be 0.20 persons per 100,000 population per year in Japan, accounting for $0.22 \%$ of all thyrotoxic patients and $5.4 \%$ of hospitalized thyrotoxic patients.

- Graves' disease (GD) was the most common cause of thyrotoxicosis among patients with TS. In $45 \%$ of cases, the duration between TS onset and the initial diagnosis of GD was less than one year. Approximately $20 \%$ of patients developed TS before they received antithyroid drug (ATD) treatment.

- Factors classically considered to trigger TS were present in 70\% of patients with TS. The most common trigger of TS was the irregular use or discontinuation of anti-thyroid medication. The second most common trigger was infection.

- The mortality rate of patients with definite or suspected TS was $10.7 \%$ (38/356). Individually, the mortality rates for definite and suspected cases were $11.0 \%$ and $9.5 \%$, respectively.

- The most common causes of death in the 356 patients with TS were multiple organ failure (MOF) and congestive heart failure (CHF).

- Disease severities as assessed using Acute Physiology and Chronic Health Evaluation (APACHE) II and Sequential Organ Failure Assessment (SOFA) scores were significantly correlated with mortality.

- Glasgow Coma Scale (GCS) score and blood urea nitrogen (BUN) level were associated with irreversible damages in survivors.

- Serum thyroid hormone concentrations were similar among patients with TS patients but no TS. Several patients with TS had normal serum fT3 levels, increased serum fT4 levels, and suppressed serum TSH levels. FT3 and the fT3/fT4 ratio inversely correlated with disease severity.

- Compared with our initial diagnostic criteria, the only change in the final version of our diagnostic criteria for TS was the addition of serum bilirubin concentration $>3 \mathrm{mg} / \mathrm{dL}$.

- Most patients $(78.1 \%)$ received methimazole (MMI). There were no significant differences in mortality or disease severity between patients treated with MMI and those receiving propylthiouracil (PTU).

- Mortality was significantly higher in patients treated with the non-selective beta-adrenergic receptor antagonists (betaAAs) propanol than in patients treated with other types of beta-AAs.

associated with thyroid hormone metabolism (deiodinases type 1 [D1], 2 [D2], and 3 [D3]), thyroid hormone transporters, and thyroid hormone receptors $(\operatorname{TR} \alpha$ and $\operatorname{TR} \beta)$. For example, activation of $\mathrm{NF} \kappa \mathrm{B}$ was shown to play an important part in the upregulation of D2 in hypothalamic tanycytes during inflammation (15). D1 is also sensitive to cytokines; D1 expression in a liver cell line decreases with interleukin $1 \beta$ stimulation, and this response can be abolished by simultaneous inhibition of $\mathrm{NF} \kappa \mathrm{B}$ and activator protein 1 (16). The liver is one of the key metabolizing organs of thyroid hormones. It expresses the thyroid hormone transporters

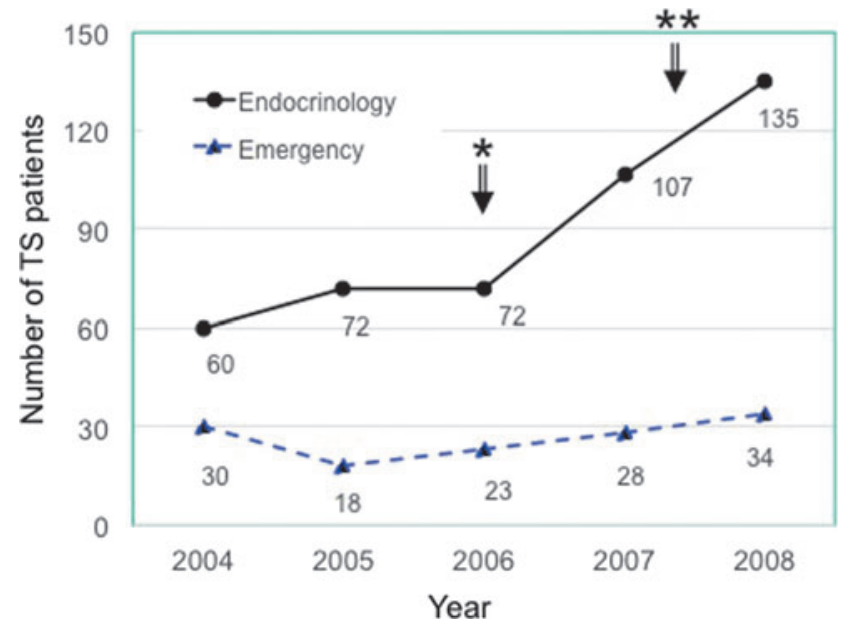

FIG. 1. Changes in the number of patients with thyroid storm (TS) managed by endocrinology and emergency departments. *Creation of Japanese task force for TS; **initial dissemination of tentative diagnostic criteria for TS. mono carboxylate transporter 8 (MCT8) and MCT10, both D1 and D3 (although D3 is expressed at very low concentrations in a healthy liver), $\operatorname{TR} \beta 1$, and TR $\alpha 1$ (14). Thus, infection is highly suspected to influence intracellular levels and metabolism of thyroid hormones in various organs and systems. Other precipitating factors including ketoacidosis, hypoxemia, and toxins are highly likely to have similar effects on the metabolism and actions of thyroid hormone.

Patient susceptibility may also affect TS development. Elderly patients may be vulnerable to thyrotoxicosis. The author's study, however, demonstrated that the mean age of

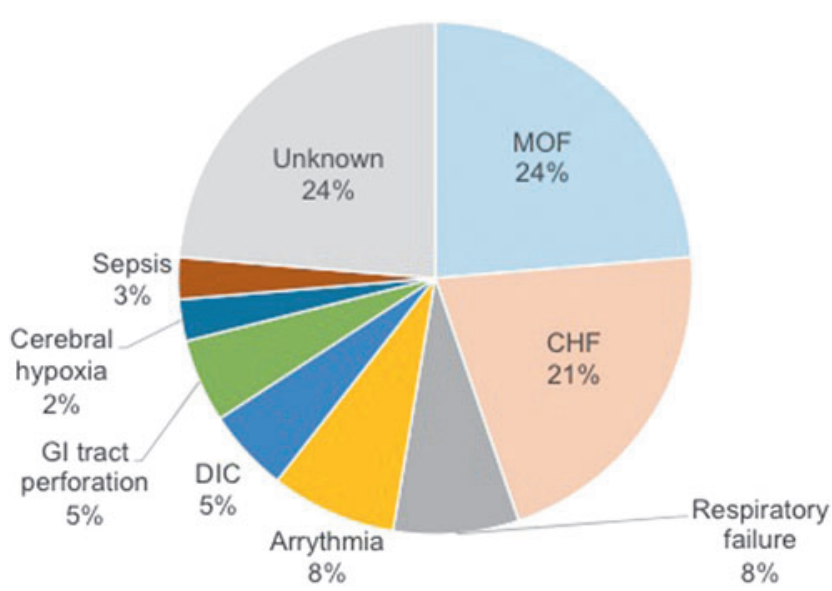

FIG. 2. Causes of death in Japanese patients with TS. $\mathrm{CHF}$, congestive heart failure; DIC, disseminated intravascular coagulation; GI, gastrointestinal; MOF, multiple organ failure. 
patients with TS was $42.1 \pm 14.9$ years (range $7-73$ years), which was not significantly different from the age of patients with uncomplicated thyrotoxicosis (10). Moreover, there was no significant difference in mortality between TS aged $\geq 65$ and $<65$ years.

\section{Clinical Manifestations of TS}

Patients with definite TS, suspected TS, and thyrotoxicosis but not TS had similar clinical manifestations. Several patients with TS had normal serum free T3 (fT3) levels, increased serum free T4 (fT4) levels, and suppressed serum thyrotropin (TSH) levels $(10,12)$.

Approximately two thirds of patients with TS had body temperatures $\geq 38.0^{\circ} \mathrm{C}$ (10). In $76.2 \%$ of patients with definite TS and $60.8 \%$ of patients with suspected TS, the heart rate was $\geq 130 \mathrm{bpm}$ and $\geq 120 \mathrm{bpm}$, respectively. Regarding central nervous system (CNS) manifestations, more than half of the patients with definite TS had abnormal GCS values $(53.5 \%)$ or Japan Coma Scale values (62.6\%). Gastrointestinal (GI) or hepatic manifestations were present in $68.3 \%$ of patients with TS. Approximately $40 \%$ of patients with TS had features of $\mathrm{CHF}$. Severe features of CHF were present in $30.9 \%$ of patients with definite TS and $18.9 \%$ patients with suspected TS. Atrial fibrillation (AF) was observed in $39.3 \%$ of patients with definite TS and $33.8 \%$ of patients with suspected TS. Of note, AF occurred in $52.6 \%$ of patients who died.

Among patients with TS, the occurrence of any one of the five major groups of clinical manifestations (CNS, fever, tachycardia, CHF, and GI or hepatic) was not positively correlated with the occurrence of any other group of clinical manifestations. The occurrence of CHF and fever was negatively correlated, including tachycardia and GI or hepatic manifestations, but the correlation coefficients were low. Among the patients with definite TS, 76\% had more than three manifestations, consistent with MOF (Fig. 3). The most common association, found in $15 \%$ of patients with definite TS, was concomitant presence of CNS symptoms, fever, tachycardia, and GI or hepatic manifestations. The next most common association, found in $11 \%$ of patients with definite TS, was the presence of CNS symptoms, tachycardia, and GI or hepatic manifestations.

\section{Diagnosis of TS}

In the mid-2000s, there were no peer-reviewed published diagnostic criteria for TS. In 1993, the criteria included in the Burch-Wartofsky Point Scale (BWPS) were reported (9). These criteria are useful, but scores allocated to signs and symptoms in this diagnostic scheme are complex and have not been validated. Therefore, an attempt was made to develop novel diagnostic criteria for TS based on patients described in the literature. Since TS is rare, a prospective study that develops criteria was considered very difficult and timeconsuming. Therefore, the criteria were mainly based on information obtained from the literature through PubMed (www.ncbi.nlm.nih.gov/pubmed) and the Ichushi databases (http://login.jamas.or.jp). Publications from 1992 to 2006 in PubMed and from 1983 to 2006 in the Ichushi database were included. Ninety-three original case reports and seven unpublished cases of TS diagnosed at the researchers' facilities were collected, and clinical parameters extracted from these cases were analyzed. Based on the analysis of these data, the first edition of diagnostic criteria for TS was created, and its incidence in Japan was surveyed (10). While developing the diagnostic criteria, a consensus decision was made that thyrotoxicosis would be considered an absolute criterion for the diagnosis of TS. Next, the prevalence of various clinical features in these patients was compared to the corresponding prevalence in patients with thyrotoxicosis but not TS. The patterns of combinations of clinical manifestations were also analyzed (Fig. 3) (10). Patterns were stratified by the presence or absence of CNS manifestations because these were most common and appeared to be very specific to TS. Since TS is a very rare disease, extremely high specificity (i.e., >99.9\%) was needed to obtain a reasonable positive predictive value (PPV; Table 3). When the incidence of TS among patients with thyrotoxicosis is estimated as $1 / 500(0.2 \%)$, which
FIG. 3. Combinations of clinical features in Japanese patients with TS. (A) $\mathrm{Pa}$ tients with CNS features $(n=238)$; (B) patients without CNS features $(n=44)$. $\mathrm{CNS}$, central nervous system; bpm, beats per minute.
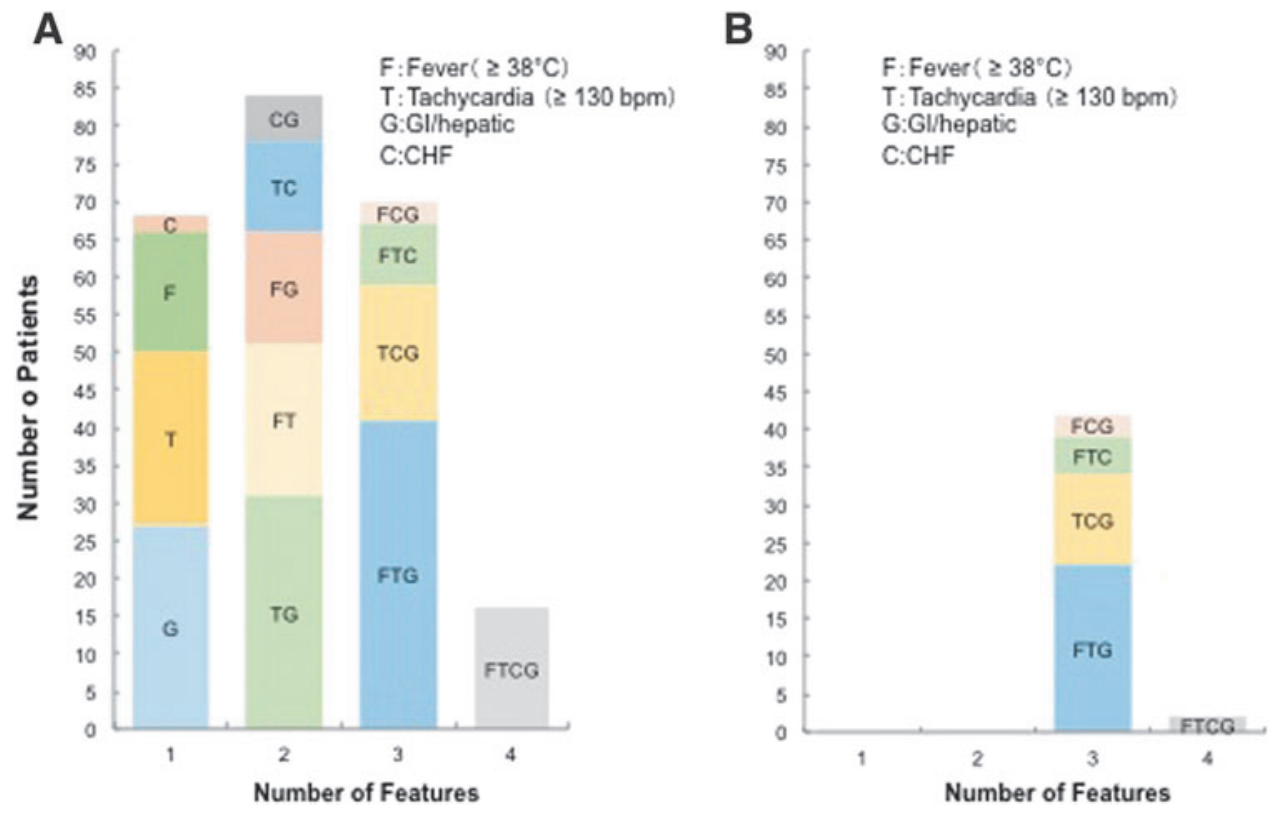
Table 3. Positive and Negative Predictive Values Based on Different Specificities And Sensitivities When the InCIDEnCE of Thyroid Storm Is Estimated as 1 Per $500(0.2 \%)$ Among PATIEnts With Thyrotoxicosis

\begin{tabular}{|c|c|c|c|c|c|c|}
\hline & & \multicolumn{2}{|c|}{ Thyrotoxicosis } & \multirow[b]{2}{*}{ Total } & & \\
\hline & & $T S$ & Non-TS & & & \\
\hline \multicolumn{7}{|c|}{ A. If both specificity and sensitivity are $80 \%$} \\
\hline TS based on the diagnostic criteria & $\begin{array}{l}\text { Yes } \\
\text { No }\end{array}$ & $\begin{array}{r}16 \\
4\end{array}$ & $\begin{array}{l}2000 \\
8000\end{array}$ & $\begin{array}{l}2016 \\
8004\end{array}$ & $\begin{array}{l}\boldsymbol{P P V} \\
N P V\end{array}$ & $\begin{array}{r}\mathbf{0 . 7 9 \%} \\
99.95 \%\end{array}$ \\
\hline Total & & 20 & 10,000 & 10,020 & & \\
\hline \multicolumn{7}{|c|}{ B. If specificity is $99.9 \%$ and sensitivity is $80 \%$} \\
\hline TS based on the diagnostic criteria & $\begin{array}{l}\text { Yes } \\
\text { No }\end{array}$ & $\begin{array}{r}16 \\
4\end{array}$ & $\begin{array}{r}10 \\
9990\end{array}$ & $\begin{array}{r}26 \\
9994\end{array}$ & $\begin{array}{l}\boldsymbol{P P V} \\
N P V\end{array}$ & $\begin{array}{l}\mathbf{6 1 . 5 4 \%} \\
99.96 \%\end{array}$ \\
\hline Total & & 20 & 10,000 & 10,020 & & \\
\hline
\end{tabular}

Bold, point of interest.

PPV, positive predictive values; NPV, negative predictive values.

turned out to be relatively accurate in the nationwide survey, diagnostic criteria with $99.9 \%$ specificity and $80 \%$ sensitivity yielded only $61.5 \%$ PPV. Diagnostic criteria with $80 \%$ specificity and $80 \%$ sensitivity were not useful (only $0.97 \%$ PPV). Considering that at least two clinical features are present for a diagnosis of TS, the specificity of each feature should be $>90 \%$. Furthermore, two grades of severity for TS, definite and suspected, were created to increase sensitivity.

After the nationwide surveys, the criteria were finalized based on the clinical features and the clinical course of these patients in the second edition of diagnostic criteria for TS (10). First, the prevalence and independence of clinical manifestations, individually and in combination, of these patients and of thyrotoxicosis without TS (Tox-NoTS) patients were examined. Next, the study investigated whether the cutoff values for each manifestation provided appropriate sensitivity, specific-

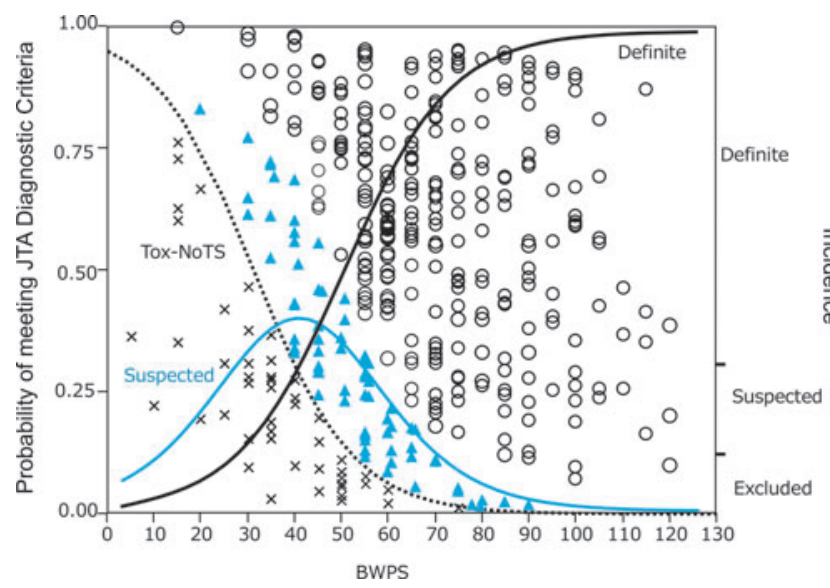

FIG. 4. Relationship between scores based on BurchWartofsky Point Scale (BWPS) and diagnosis based on criteria for TS from the Japan Thyroid Association (JTA) (10). Tox-NoTS, thyrotoxicosis without TS. $\bigcirc$, definite cases; $\boldsymbol{\Delta}$, suspected cases; $\times$, Tox-NoTS. Curved lines indicate the probabilities of JTA criteria by BWPS based on logistic regression analysis (black solid line, definite cases; blue solid line, suspected cases; black dotted line, Tox-NoTS). ity, and positive and negative predictive values to differentiate between definite TS, suspected TS, and Tox-NoTS. Based on these analyses, only one change was made to generate the final diagnostic criteria for TS. This change was related to serum bilirubin concentration. The mortality rate for definite and suspected TS patients with total serum bilirubin concentration $>3.0 \mathrm{mg} / \mathrm{dL}$ was significantly higher than the rate in patients with lower serum bilirubin concentrations. Therefore, a serum total bilirubin concentration of $>3 \mathrm{mg} / \mathrm{dL}$ was included as a criterion for GI or hepatic manifestations, which was the only difference between the two versions.

The author's criteria for the diagnosis of TS differ in two major ways from the BWPS criteria. First, thyrotoxicosis is implied in the BWPS criteria, but it is clearly stated in the author's criteria that evidence of thyrotoxicosis is a prerequisite for the diagnosis of TS. It was felt that making thyrotoxicosis a prerequisite is needed to reduce false-positives. Second, in contrast to the BWPS, there is no scoring system for the author's criteria for TS. It is impossible, in the author's opinion, to generate a scoring system in a retrospective study, since a prospective study is needed for validation. Indeed, the BWPS score is neither evidence-based nor validated. In addition, the BWPS scoring system is rather complex. Although there was a significant correlation between the author's diagnostic criteria and the BWPS $\left(r^{2}=0.277 ; p<\right.$ $0.0001)$, the fact that its contribution to the diagnosis by the author's criteria was small indicates a discrepancy between the two diagnostic systems (Fig. 4). Notwithstanding such a discrepancy, however, both the BWPS and the author's criteria may be helpful in diagnosing TS. All of the author's criteria for TS require elevated serum thyroid hormone levels, fT3 and/or fT4, as a prerequisite item. It is, however, well known that circulating thyroid hormones, particularly T3, tend to be relatively low in severe non-thyroid illness. This is the case in TS. In fact, several patients with definite TS had normal serum fT3 levels, despite elevated serum fT4 levels and low serum TSH levels. It should be kept in mind that even serum fT4 and TSH levels are decreased in severe illness. In addition, the administration of drugs, including ATDs, iodine, and corticosteroids, modifies thyroid function test results. Therefore, there may be a need to expand the diagnostic criteria for TS, but further studies will be needed. 


\section{Severity and Prognosis of TS}

This study sought to determine the severity of illness in patients with TS, or the perceived severity, based on three indexes: the level of inpatient care they were assigned in an intensive care unit, a high care unit, or a general ward; their Acute Physiology and Chronic Health Evaluation (APACHE) II score (17); and the Sequential Organ Failure Assessment (SOFA) score $(18,19)$. The means $\pm S D$ for APACHE II and SOFA scores were 10.97 \pm 0.35 (range $=$ $0-37$ ) and $2.67 \pm 0.47$ (range $0-12)$, respectively $(n=354)$. Patients who died had significantly higher scores than those who survived: died versus survived $=15.00 \pm 1.04$ versus $10.48 \pm 0.36(p<0.0001)$ for APACHE II and 3.12 \pm 0.51 versus $2.38 \pm 0.13(p<0.0001)$ for SOFA, respectively.

The mortality rate for TS was $10.7 \%$, lower than previously reported $(4,20)$. Improvements in the general management of patients and early diagnosis likely contributed to the lower mortality rate. The most frequent direct causes of death, such as shock, DIC, and MOF, were very similar in the definite and suspected groups. This observation strongly suggests that mortality depends on the severity of complications rather than the characteristic clinical features of thyroid crisis. In addition, treatments or interventions for severe conditions, which were variably used among physicians, probably influenced the mortality rate. An association between mortality and a CNS manifestation or CHF was consistently observed in univariate regression analysis. Indeed, TS mortality has been attributed to these factors in the literature $(4,21)$. However, in the multiple regression analysis, these were not significant independent factors for patient death. This is perhaps because recent developments in the management of critically ill patients reduced mortality related to these factors. However, CNS symptoms did have a profound impact on the quality of life, as shown by the analysis of irreversible damage (10). In general, many factors were evaluated that are typically assessed in critically ill patients. It is recommended that all of these complications be carefully managed in the treatment of TS.

\section{Management and Treatment of TS}

Recent nationwide surveys in Japan have revealed that mortality for TS remains $>10 \%$ (10). Even when patients survive, some have irreversible damage. Therefore, the prognosis of patients with TS needs to be improved. Since MOF is characteristic of TS, multidisciplinary care involving endocrinologists, cardiologists, neurologists, and hepatologists is necessary for management. Furthermore, the decompensated state associated with TS often requires comprehensive, highly advanced medical treatment (11). Although several textbooks and guidelines have described the treatment of TS (22-25), nationwide surveys in Japan revealed that methimazole (MMI) was preferentially used in TS, despite recommendations for the use of propylthiouracil (PTU) (12). Therefore, more updated guidelines for the management of TS need to be established in Japan and other countries. Such guidelines should be helpful to many practitioners. Indeed, after the diagnostic criteria and data from nationwide surveys about TS were reported (10), information on therapeutic interventions and management became eagerly anticipated $(22,26,27)$.

Five treatment targets are important in TS: (i) control of thyrotoxicosis, (ii) control of systemic symptoms and signs, (iii) control of organ-specific manifestations, (iv) identification and therapy of triggers, and (v) definitive therapy of thyrotoxicosis (11). Although the appropriate responses to these issues have been described in endocrinology textbooks and reviews, several clinical questions remain such as: (i) the choice and route of administration for ATDs, (ii) the timing of iodide therapy, (iii) the criteria to judge the severity of TS, and (iv) the choice and fine-tuning of treatment based on severity and pathophysiological state. Although beta-adrenergic receptor antagonists (beta-AAs) are often used to treat TS, inappropriate agent choice or dose may lead to worse outcomes in patients with severe heart failure (12). Furthermore, TS is characterized by MOF, decompensation, and highly variable clinical presentation, a clinical picture that requires comprehensive treatment. TS is an emergency disorder characterized by rapid deterioration. Therefore, an algorithm-based approach is useful for the management of TS (11).

Given this context, an attempt was made to create recommendations for the management of TS based on the following principles (11). Recommendations should (i) contain information on both the diagnosis and treatment of TS; (ii) illustrate algorithms; (iii) consider the severity and pathophysiology of TS; (iv) be detailed, concrete, and useful in clinical practice; (v) be evidence-based; and (vi) possibly be internationally applicable. Based on the evidence obtained from nationwide surveys (10) and additional literature reviews, guidelines were established for the management of TS in 2016 (11). The guidelines include 15 recommendations for the treatment of thyrotoxicosis and organ failure in the CNS, cardiovascular system, the liver, and the GI tract; criteria for admission to the intensive care unit; and prognostic evaluation. Approaches to prevent TS and roles for definitive therapy were also proposed. Because of space limitations, only the treatments for hyperthyroidism due to GD, tachycardia, and CHF are mentioned here. Treatments for other features can be found in the full guideline paper (11).

For thyrotoxicosis due to GD, a multimodality approach with ATDs, inorganic iodide, corticosteroids, beta-AAs, and antipyretic agents should be used to ameliorate thyrotoxicosis and its adverse effects on multiple organ systems. ATDs, either MMI or PTU, should be used for the treatment of hyperthyroidism in TS. Although the main action of ATDs is to inhibit thyroid peroxidase directly by coupling iodotyrosine to thyroglobulin molecules, the major functional difference between MMI and PTU is that large doses of PTU ( $\geq 400 \mathrm{mg} /$ day) inhibit type I deiodinase activity in the thyroid gland and other peripheral organs, which may therefore acutely decrease T3 levels more than MMI $(28,29)$. These are the reasons that PTU, rather than MMI, is recommended in the guidelines issued by the American Thyroid Association (ATA) (25). A nationwide survey performed by the JTA revealed that both fT3 levels and the fT3/fT4 ratio, but not fT4 levels, were inversely correlated with disease severity as assessed by APACHE II and SOFA scores in patients with TS (12). These findings strongly suggest that the conversion of T4 to T3 could already be suppressed in severe TS. In addition, there were no significant differences in disease severity or mortality between patients with TS treated with MMI or PTU (12). Therefore, like PTU, MMI may be useful during severe TS with reduced T4-to-T3 conversion. Regarding the long-term efficacy of ATDs in thyrotoxicosis in compensated GD, a randomized prospective study performed in Japan 
revealed that MMI $(30 \mathrm{mg} /$ day) normalized thyroid hormone levels more rapidly than PTU $(300 \mathrm{mg} /$ day). This study also showed that the incidence of adverse effects in patients treated with MMI was significantly lower than in those treated with PTU (30). Based on these findings, the JTA guidelines recommend MMI as the first-choice ATD for the treatment of compensated GD except during early pregnancy (31). Therefore, MMI has been favored by physicians in Japan for the treatment of compensated GD and has been used more frequently to treat uncompensated thyrotoxicosis in TS, according to the nationwide surveys (278/356 patients; 78\%) (12). In addition, a recent study from the United States also reported no significant differences in outcomes between patients with TS treated with MMI or PTU (32). Taken together, these observations provide supporting evidence that MMI may not be disadvantageous compared to PTU for the treatment of thyrotoxicosis in TS. On the other hand, PTU may be useful in cases where elevated T3 levels are the dominant feature.

Inorganic iodide should be administered concurrently with ATDs to patients with TS caused by thyrotoxic diseases associated with hyperthyroidism. Several textbooks $(8,24)$ and the ATA guidelines (25) recommend that inorganic iodide be administered only after about an hour after the administration of ATDs to prevent the organification of iodide. A recent prospective study comparing MMI with MMI plus potassium iodide (KI) in terms of rapid normalization of thyroid hormones in compensated GD reported that fT3 levels in the combination treatment group decreased significantly faster than in the MMI group. None of the patients showed an increase in thyroid hormone levels or disease aggravation during combination treatment (33). Another prospective study evaluating the efficacy of MMI $15 \mathrm{mg} /$ day plus inorganic iodide $38.2 \mathrm{mg} /$ day (equivalent to $50 \mathrm{mg} \mathrm{KI}$; M15 + KI) versus MMI $30 \mathrm{mg} /$ day (M30) for the treatment of moderate to severe uncomplicated GD also demonstrated that combined treatment with M15 + KI improved fT4 levels significantly faster than treatment with M30, with no exacerbation of thyrotoxicosis in patients treated with M15 + KI (34). Based on these findings from two large prospective trials performed in Japan, it is recommended that large doses of inorganic iodide be administered concurrently with ATDs to patients with GD complicated with TS. Indeed, before ATDs were developed, administration of inorganic iodide was the most reliable treatment for TS (2).

Beta1-selective AAs such as landiolol, esmolol (intravenous), or bisoprolol (oral) are the first-line agents to treat tachycardia in TS (11). Other oral beta1-selective drugs are also recommended. Although the non-selective beta-AA propranolol is not contraindicated, it is not recommended for the treatment of tachycardia in TS with CHF; 4/12 patients with Killip class IV CHF treated with non-selective beta-AAs died (12). In addition, the use of propranolol without landiolol significantly contributed to increased mortality in these patients $\left(\mathrm{OR}=7.6381 ; r^{2}=0.2189\right.$; $p=0.0386$ ). The use of non-selective beta-AAs, particularly propanol, requires caution.

\section{Future Directions}

Several clinical questions remain, including the use of PTU versus MMI and simultaneous versus sequential use of ATDs and iodine. To answer these questions, a prospective prognostic study of these recommendations is currently planned. It is hoped that improved outcomes in TS will be confirmed through effective implementation of these recommendations. Furthermore, the guidelines may be revised based on the findings of this future study.

Importantly, the pathogenesis leading to the development of TS has not yet been clarified. Investigation of the mechanisms underlying MOF and decompensation is eagerly awaited because it will certainly help to improve the understanding of the characteristic features of TS and the development of novel therapies for TS.

\section{Acknowledgments}

I deeply thank the members of the JTA and Japan Endocrine Society task force for the establishment of diagnostic criteria and nationwide surveys for TS (Dr. Tetsurou Satoh, Dr. Osamu Isozaki, Dr. Shu Wakino, Dr. Atsushi Suzuki, Dr. Tadao Iburi, Dr. Kumiko Tsuboi, Dr. Naotetsu Kanamoto, Dr. Hajime Otani, Dr. Yasushi Furukawa, and Dr. Satoshi Teramukai). I also thank the members of JTA and Japan Endocrine Society, as well as participating doctors from Japanese hospitals and clinics, for their valuable and kind cooperation in the questionnaires and nationwide surveys. This study was supported by a fund from the Ministry of Health, Labor, and Welfare of Japan.

\section{Author Disclosure Statement}

The author has nothing to disclose, except for research grants from the Ministry of Health, Labor, and Welfare of Japan.

\section{References}

1. Lahey F 1928 Thyroid crisis (the crisis of exophthalmic goiter). N Engl J Med 199:255-257.

2. Greene EI, Greene JM 1932 Thyroid crisis. Ann Surg 95:537-543.

3. Rosenberg IN 1970 Thyroid storm. N Engl J Med 283: 1052-1053.

4. Tietgens ST, Leinung MC 1995 Thyroid storm. Med Clin North Am 79:169-184.

5. Cooper DS 2012 Treatment of thyrotoxicosis. In: Braverman L, Cooper DS (eds) Werner \& Ingbar's The Thyroid. A Fundamental and Clinical Text. Tenth edition. Lippincott Williams \& Wilkins, Philadelphia, PA, pp 492-516.

6. Sarlis NJ, Gourgiotis L 2003 Thyroid emergencies. Rev Endocr Metab Disord 4:129-136.

7. Migneco A, Ojetti V, Testa A, De Lorenzo A, Gentiloni Silveri N 2005 Management of thyrotoxic crisis. Eur Rev Med Pharmacol Sci 9:69-74.

8. Wartofsky L 2005 Thyrotoxic storm. In: Braverman L, Utiger R (eds) Werner \& Ingbar's The Thyroid. Ninth edition. Williams \& Wilkins, Philadelphia, PA, pp 651657.

9. Burch HB, Wartofsky L 1993 Life-threatening thyrotoxicosis. Thyroid storm. Endocrinol Metab Clin North Am 22:263-277.

10. Akamizu T, Satoh T, Isozaki O, Suzuki A, Wakino S, Iburi T, Tsuboi K, Monden T, Kouki T, Otani H, Teramukai S, Uehara R, Nakamura Y, Nagai M, Mori M; Japan Thyroid Association 2012 Diagnostic criteria, clinical features, and 
incidence of thyroid storm based on nationwide surveys. Thyroid 22:661-679.

11. Satoh T, Isozaki O, Suzuki A, Wakino S, Iburi T, Tsuboi K, Kanamoto N, Otani H, Furukawa Y, Teramukai S, Akamizu T 20162016 Guidelines for the management of thyroid storm from The Japan Thyroid Association and Japan Endocrine Society (First edition). Endocr J 63:1025-1064.

12. Isozaki O, Satoh T, Wakino S, Suzuki A, Iburi T, Tsuboi K, Kanamoto N, Otani H, Furukawa Y, Teramukai S, Akamizu T 2016 Treatment and management of thyroid storm: analysis of the nationwide surveys: the taskforce committee of the Japan Thyroid Association and Japan Endocrine Society for the establishment of diagnostic criteria and nationwide surveys for thyroid storm. Clin Endocrinol 84:912-918.

13. Dillmann WH 1997 Thyroid storm. Curr Ther Endocrinol Metab 6:81-85.

14. Fliers E, Bianco AC, Langouche L, Boelen A 2015 Thyroid function in critically ill patients. Lancet Diabetes Endocrinol 3:816-825.

15. Pang XP, Hershman JM, Mirell CJ, Pekary AE 1989 Impairment of hypothalamic-pituitary-thyroid function in rats treated with human recombinant tumor necrosis factoralpha (cachectin). Endocrinology 125:76-84.

16. Kwakkel J, Wiersinga WM, Boelen A 2006 Differential involvement of nuclear factor-kappaB and activator protein-1 pathways in the interleukin-1beta-mediated decrease of deiodinase type 1 and thyroid hormone receptor beta1 mRNA. J Endocrinol 189:37-44.

17. Wagner DP, Draper EA 1984 Acute physiology and chronic health evaluation (APACHE II) and Medicare reimbursement. Health Care Financ Rev Suppl:91-105.

18. Vincent JL, Moreno R, Takala J, Willatts S, De Mendonca A, Bruining H, Reinhart CK, Suter PM, Thijs LG 1996 The SOFA (Sepsis-related Organ Failure Assessment) score to describe organ dysfunction/failure. On behalf of the Working Group on Sepsis-Related Problems of the European Society of Intensive Care Medicine. Intensive Care Med 22:707-710.

19. Antonelli M, Moreno R, Vincent JL, Sprung CL, Mendoca A, Passariello M, Riccioni L, Osborn J 1999 Application of SOFA score to trauma patients. Sequential Organ Failure Assessment. Intensive Care Med 25:389-394.

20. Burger AG, Philippe J 1992 Thyroid emergencies. Baillieres Clin Endocrinol Metab 6:77-93.

21. Gavin LA 1991 Thyroid crises. Med Clin North Am 75:179-193.

22. Wartofsky L 2012 Clinical criteria for the diagnosis of thyroid storm. Thyroid 22:659-660.

23. Nayak B, Burman K 2006 Thyrotoxicosis and thyroid storm. Endocrinol Metab Clin North Am 35:663-686, vii.

24. Davies T, Laurberg P, Bahn RS 2015 Thyroid storm. In: Melmed S, Polonsky K, Larsen P, Kronenberg H (eds)
Williams Textbook of Endocrinology. Thirteenth edition. Elsevier Saunders, Philadelphia, PA, pp 393-395.

25. Ross DS, Burch HB, Cooper DS, Greenlee MC, Laurberg P, Maia AL, Rivkees SA, Samuels M, Sosa JA, Stan MN, Walter MA 20162016 American Thyroid Association guidelines for diagnosis and management of hyperthyroidism and other causes of thyrotoxicosis. Thyroid 26:1343-1421.

26. Feldt-Rasmussen U, Emerson $\mathrm{CH} 2012$ Further thoughts on the diagnosis and diagnostic criteria for thyroid storm. Thyroid 22:1094-1095.

27. Horvath K 2014 Cover story: gathering storm. Endocrine News. Endocrine Society, Washington, DC, pp 10-13.

28. Abuid J, Larsen PR 1974 Triiodothyronine and thyroxine in hyperthyroidism. Comparison of the acute changes during therapy with antithyroid agents. J Clin Invest 54:201-208.

29. Maia AL, Kim BW, Huang SA, Harney JW, Larsen PR 2005 Type 2 iodothyronine deiodinase is the major source of plasma T3 in euthyroid humans. J Clin Invest 115:25242533.

30. Nakamura H, Noh JY, Itoh K, Fukata S, Miyauchi A, Hamada N 2007 Comparison of methimazole and propylthiouracil in patients with hyperthyroidism caused by Graves' disease. J Clin Endocrinol Metab 92:2157-2162.

31. Japan Thyroid Association 2011 Selection of Antithyroid Agents. Therapeutic Guideline for Graves' Disease 2011. Nankodo, Inc., Tokyo, Japan, pp 24-30.

32. Vydro L, Joglekar S, Sheh S, Yau H, Naing S 2015 Choice of antithyroid drugs and the outcome of patients with thyroid storm. 15th International Thyroid Congress, Orlando, FL.

33. Takata K, Amino N, Kubota S, Sasaki I, Nishihara E, Kudo T, Ito M, Fukata S, Miyauchi A 2010 Benefit of short-term iodide supplementation to antithyroid drug treatment of thyrotoxicosis due to Graves' disease. Clin Endocrinol 72:845-850.

34. Sato S, Noh JY, Sato S, Suzuki M, Yasuda S, Matsumoto M, Kunii Y, Mukasa K, Sugino K, Ito K, Nagataki S, Taniyama M 2015 Comparison of efficacy and adverse effects between methimazole $15 \mathrm{mg}+$ inorganic iodine $38 \mathrm{mg} /$ day and methimazole $30 \mathrm{mg} /$ day as initial therapy for Graves' disease patients with moderate to severe hyperthyroidism. Thyroid 25:43-50.

Address correspondence to: Takashi Akamizu, MD, PhD The First Department of Medicine Wakayama Medical University 811-1 Kimi-idera Wakayama 641-8509 Japan

E-mail: akamizu@wakayama-med.ac.jp 